\title{
A Research for Implementing Image Interpolation Using Inpainting and Shearlet Transform
}

\author{
P.J. Sajith, S.U. Aswathy, Bibin Vincent, R.S. Anoop Sreekumar
}

\begin{abstract}
This paper proposes a way to improve the compression ratio of images by expunging some parts of the image prior transmission. The remaining data besides essential details for recovering the removed regions are encoded to produce the final data. At the decoding side an inpainting method is applied to retrieve the removed region. The Shearlet Transform is used for the smoothing purpose of the recovered image. This transform can identify the location of singularities of a function and also the orientation of discontinuity curves. The Shearlet Transform has the ability to provide a very accurate geometrical characterization of general discontinuity occurring in images.
\end{abstract}

Keywords--- Image Inpainting, Image Compression, Compression Ratio, Peak Signal-to-Noise Ratio, Structural Similarity.

\section{INTRODUCTION}

Each image is composed of discrete points called pixels. The value relevant to each pixel is the result of sampling from light or color intensity in the original image domain. Natural images consist of separate areas indicating the object surfaces or sceneries. Since the light intensity and color in such areas are approximately constant the relevant values for pixels are highly correlated. Every pixels in such areas are likely to be of the same or very close value compared with the adjacent pixels. Representing the image by storing all pixel values results in a large amount of redundancy.

Image in painting is a technique of filling in the missing region of an image. It is the art of modifying an image in a form that is not easily detectable by an ordinary observer. The main usage of in painting is the restoring of damaged part of the picture [1]. Without transmitter sending entire picture the receiver can recover the missing part of the image to come up with the whole image in the end. Compression is acceptable for natural images as a large amount of redundancy is included in such images. By not sending significant portion of the image that can later be restored from remaining part the amount of bits needed to transmit the image can be reduced significantly.

For the in painting method to be successful it is important to choose and erase the block that can be easily restored.

Manuscript received September 16, 2019.

P.J. Sajith, Asst. Professor, Department of Computer Science and Engineering, Vijnan Institute of Science and Technology, Ernakulam, Kerala, India.

Dr.S.U. Aswathy, Professor, Department of Computer Science and Engineering, Mangalam College of Engineering, Ettumanoor, Kottayam, Kerala India.

Dr. Bibin Vincent, Professor, Department of Computer Science and Engineering, Mangalam College of Engineering, Ettumanoor, Kottayam, Kerala India.

Dr.R.S. Anoop Sreekumar, Asst. Professor, Department of Computer Science, Malankara Catholic College, Mariagiri, Kanyakumari District, Tamil Nadu, India.
There are two types of regions that can be relatively easily reconstructed, structure and texture. It is important to properly classify these blocks into either of these two. In image in painting the only information available for reconstruction is the average value of the erased block.

The most significant information within an image is located in the boundary regions or edges. The boundary region not only specifies its overall shape but also shows how pixel values change from neighboring regions to the inner regions. So it is possible to retrieve the inner areas using pixels located on the boundaries. Therefore boundaries or edges are all the required information for displaying an image. The variation in values of pixels orthogonal to the edges is significant. Hence, areas in the neighborhood of edges may be considered as essential image information. While moving along the edge direction, no significant changes in pixel values will be observed. Moving further to the inner points of the boundaries will result in a considerable correlation for pixel values. Edges also represent some other necessary information including shapes. Redundancies related to the correlation along the edge direction may also be exploited via extracting shape information from pixel values in boundary regions.

Pixel values at the endpoints of an edge will be used for recovering the entire edge and boundary region. In order to recover boundary regions and pixels located perpendicular to the edge direction, samples of source points should be provided. These samples should come from different areas at each side of the edge.

\section{IMAGE INPAINTING}

Image inpainting is a method for recovering regions in images whose pixels are distorted or removed in some way. Inpainting methods are commonly based on partial differential equations. The method proposed in this paper is based on eliminating the information of correlated regions and filling in the missing areas using sample pixels. In this method, some regions are intentionally removed at the encoder and recovered using an inpainting or interpolation technique at the decoder. In partial differential equation techniques, pixel values around the region to be inpainted are considered to be the boundary condition for a boundary value problem. Then, a proper equation for interpolating in that area will be solved. Image inpainting has a variety of applications such as text and object removal [2], [3], denoising, super resolution, digital zooming [4], filling-in [5] and compression.

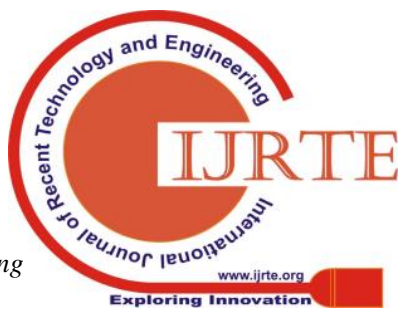




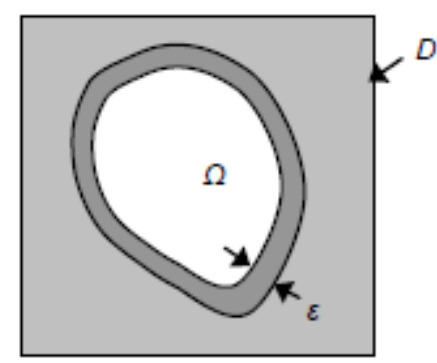

Fig. 1: A General Case of Image Inpainting Problem Image inpainting is aimed to fill in missing regions or to modify damaged regions in a visually plausible and nondetectable way. In order to well clarify the inpainting problem, assume $\mathrm{u} 0$ as the intensity function of the image defined in domain $\mathrm{D}$.

As indicated in Fig. 1, there is a hole $\Omega C D$ with unknown information. The objective is to find the recovered version of $\mathrm{u} 0$, namely $\mathrm{u}$, in such a way that the intensity function in the area $\mathrm{D}-\Omega$ remains equal to $\mathrm{u} 0$ while meaningfully filling in other regions. In this way, information on the boundary $\varepsilon$ is diffused into $\Omega$ via a $2 \mathrm{D}$ interpolation technique.

In a general form, no information is available about the regions to be inpainted. The resulting inpainted image is not necessarily similar to the original one. For the application of compression, it is necessary for the inpainted image to be similar to the original one with a sufficient degree of accuracy. As the original image is in hand, it is possible to extract all of the information required for compression with an acceptable quality. Here the only essential information for retrieving an image includes source point pixels and edges.

\section{TEXTURE INPAINTING}

Texture inpainting is to find best match from referable surrounding blocks in statistical aspect. Texture synthesis process is like below. At first, when we call texture synthesis function, we give referable neighborhood block information.

Secondly, we set the template which is $3 \times 3$ or $4 \times 4$ and next to the missed pixel we want to fill. We classify blocks whose statistical properties are similar to those of surrounding blocks into texture. So, when we want to fill the texture block, we exploit the statistical similarity with other blocks.

In big picture $(512 \times 512), 8 \times 8$ block is not a big portion. And, in smooth area, we don't use texture synthesis because of peak signal-to-noise ratio (PSNR) quality, even though texture synthesis is faster than structure inpainting. In most cases, we use texture synthesis in very coarse area or pattern area.

From those reasons, we copy the whole block $(8 \times 8)$ to the missed texture block, after finding the block which has the closest mean and variance to missed texture block. In this case, we can get a good result in visual aspect, despite of almost same result in PSNR.

\section{STRUCTURE INPAINTING}

Structure is the region that can be clearly divided into two or more sectors through clear edges. Each sector is relatively devoid of minute details and the missing block within the sector is easily predicted from surrounding blocks. Structure inpainting is the process of gradually propagating the information contained in the surrounding blocks into the missing block. Basically, this process is very similar to that of diffusion. When the heat source (surrounding block) is placed around closed area (missing block), heat (information) gradually flows into the area.

\section{SHEARLET TRANSFORM}

It is now widely acknowledged that traditional wavelet methods do not perform as well with multidimensional data. Indeed wavelets are very efficient in dealing with point wise singularities only.

In higher dimensions, other types of singularities are usually present or even dominant and wavelets are unable to handle them very efficiently. Images, for example, typically contain sharp transitions such as edges, and these interact extensively with the elements of the wavelet basis.

As a result, many terms in the wavelet representation are needed to accurately represent these objects. In order to overcome this limitation of traditional wavelets, in this paper, a new wavelet transform is introduced, namely shearlet.

\section{Continuous Shearlet Transform}

The continuous shearlet transform is a non isotropic version of the continuous wavelet transform with a superior directional sensitivity. In dimension $n=2$, this is defined as the mapping,

$$
\mathrm{SH}_{\psi} \mathrm{f}(\mathrm{a}, \mathrm{s}, \mathrm{t})=\left(\mathrm{f}, \psi_{\mathrm{a}, \mathrm{s}, \mathrm{t}}\right)
$$

Each analyzing elements $\psi$ a,s,t called shearlets has a frequency support on a pair of trapezoids, at various scales, symmetric with respect to the origin and oriented along a line of slope s. The support becomes increasingly thin as a $\rightarrow 0$.

As a result, the shearlets form a collection of well localized waveforms at various scales, orientations and locations, controlled by $\mathrm{a}, \mathrm{s}$, and $\mathrm{t}$ respectively. The frequency supports of some representative shearlets are illustrated in Fig.2

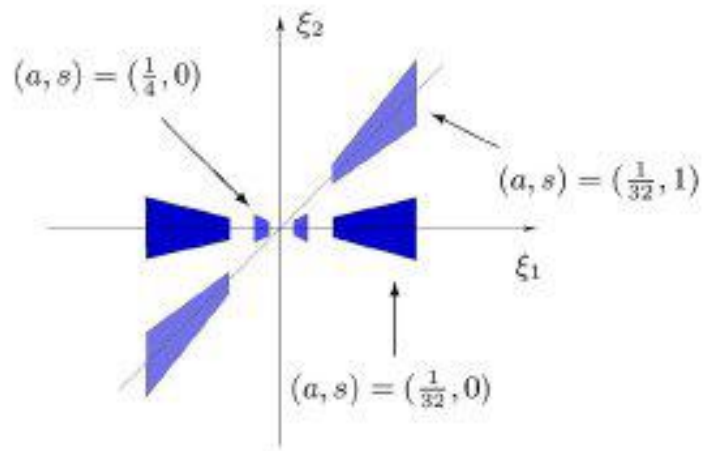

Fig. 2: Frequency Support of Shearlets for Various Values of $A$ and $S$

\section{Discrete Shearlet Transform (DST)}

By sampling the continuous shearlet transform on appropriate discretization's of the scaling, shear, and

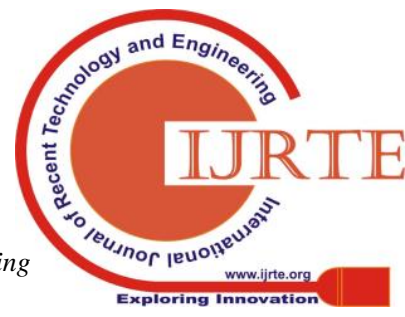


translation parameters a, $\mathrm{s}, \mathrm{t}$ one obtains a discrete transform The following procedure describes the construction of Shearlet transform and the procedure is illustrated on Fig. 3. Apply the laplacian pyramid scheme to decompose $\mathrm{fa}^{\mathrm{j}-1}$ into a low pass image $\mathrm{fa}^{\mathrm{j}}$ and a high pass image $\mathrm{fd}^{\mathrm{j}}$.

1. Compute $\mathbf{P} \mathrm{fd}^{\mathrm{j}}$ on a pseudo polar grid.

2. Apply a band pass filtering to the matrix $\mathrm{P} \mathrm{fd}^{\mathrm{j}}$.

3. Directly re-assemble the Cartesian sampled values and apply the inverse two-dimensional Fast Fourier Transform (FFT).

\section{EXPERIMENTAL RESULT}

The steps of compression are depicted in Fig. 4. The original image $\mathrm{I}$ is analyzed and the blocks to be removed with masked image to decoder. $\mathrm{R}$ should contain the locations of blocks removed and the algorithm to be used to fill in the missing region.

For missing region, fill in DC values to minimize the size of JPEG-encoded image. $\mathrm{R}$ is compressed using lossless encoder while DC-filled image D is encoded by JPEG. On the decoder side, R' and D' are decoded and R is used to fill in the removed blocks of $\mathrm{D}$.

The bit rate is calculated by (size of $\left.D^{\prime}\right)+($ size of R' (entropy encoded R)) / (size of the image). The different images are shown in Fig. 5.

For comparison purpose the PSNR values are computed and the values are tabulated and shown in Table1.

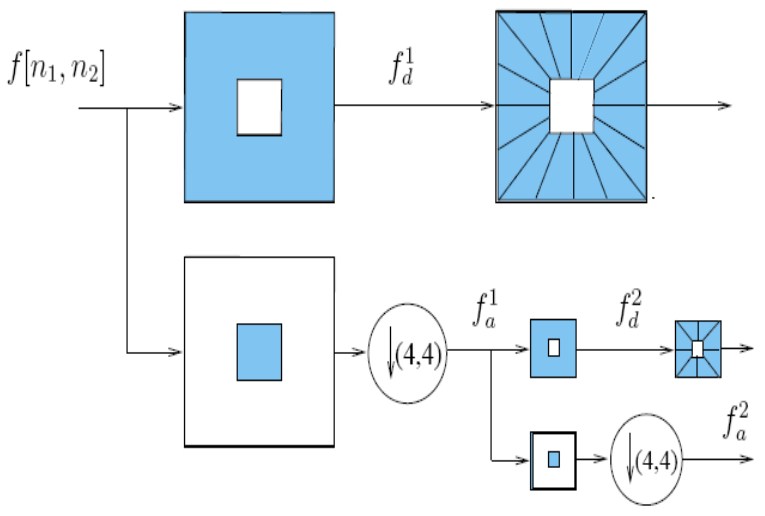

Fig. 3: Succession of Laplacian Pyramid and Directional Filtering

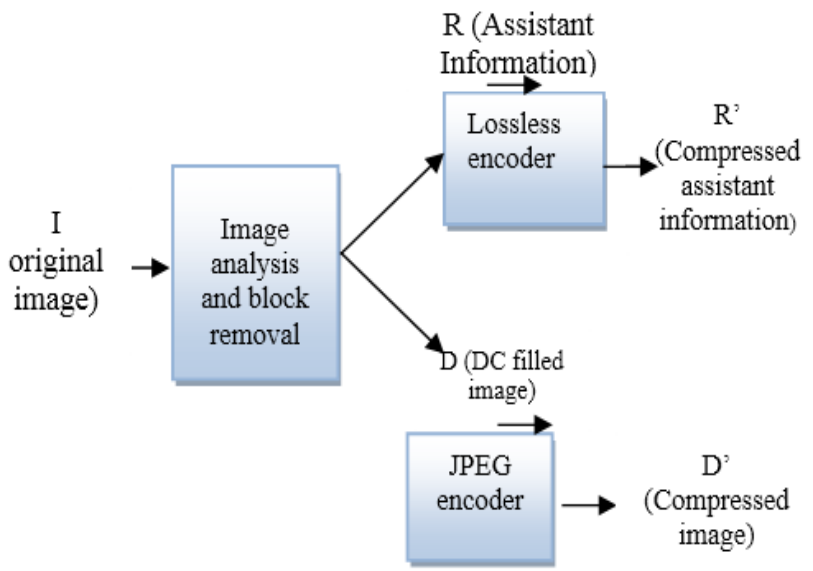

Encoder which is associated to a Parseval (tight) frame for $L^{2}\left(R^{2}\right)$. are determined. The assistant information $\mathrm{R}$ should be sent

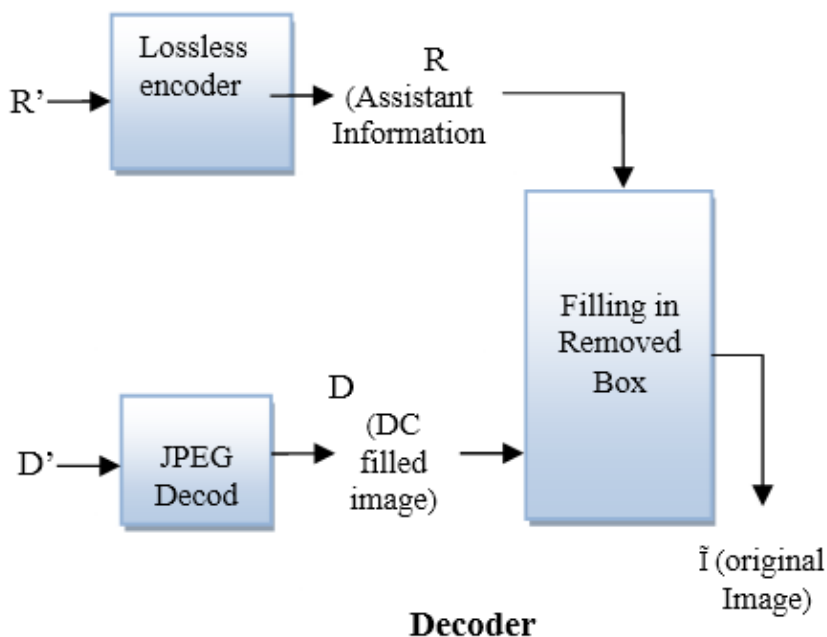

Fig. 4: An Algorithm for Image Compression Using Inpainting

Fig. 5: Performance Comparison Using PSNR Values

\begin{tabular}{|l|l|l|l|l|}
\hline \multirow{2}{*}{ Image } & \multicolumn{2}{|l|}{ Existing Method } & \multicolumn{2}{l|}{$\begin{array}{l}\text { Proposed Method } \\
\text { Sheatlet }\end{array}$} \\
\cline { 2 - 5 } & $\boldsymbol{S S I M}$ & $\begin{array}{l}\boldsymbol{P S N R} \\
(\boldsymbol{d B})\end{array}$ & $\boldsymbol{S S I M}$ & PSNR $(\boldsymbol{d B})$ \\
\hline Image 1 & 0.8965 & 29.24 & 0.9272 & 36.45 \\
\hline Image 2 & 0.8864 & 33.67 & 0.9028 & 40.67 \\
\hline Image 3 & 0.8137 & 31.45 & 0.8798 & 37.45 \\
\hline
\end{tabular}

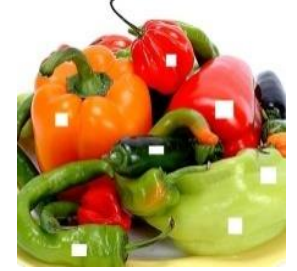

(a)

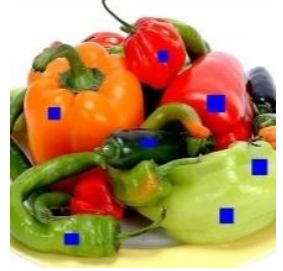

(b)

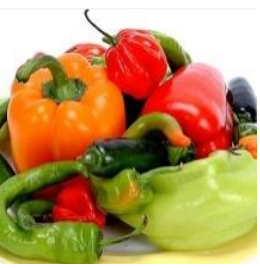

(c)
Fig. 6: (a) Noisy ima\$ge (b) Masked Image (c) Received Image after Inpainting

A conventional image quality index is the PSNR, which is the ratio between the maximum possible power of a signal and the power of the corrupting noise that affects the fidelity of its representation.

It is widely used for the estimation of quality in lossy image compression algorithms. The signal in this case is the original data and the noise is the error introduced by compression. This index is popular for its simplicity; however, it loses its advantages compared with natural human perception [6].

A better index for image quality measurement is the structural similarity (SSIM), which is a method for measuring the similarity between two images. The SSIM index is a full reference metric, the measuring of image quality based on an initial uncompressed image as a reference and is calculated as,

$$
\mathrm{SSIM}=\frac{4 \sigma_{X Y} \mu_{X} \mu_{Y}}{\left(\sigma_{X}^{2}+\sigma_{Y}^{2}\right)\left(\mu_{X}^{2}+\mu_{Y}^{2}\right)}
$$

where, $\sigma_{\mathrm{XY}}$ the covariance of $\mathrm{X}$ and $\mathrm{Y}, \mu_{\mathrm{X}}$ the average of $X, \mu_{Y}$ the average of $Y, \sigma_{X}^{2}$ the variance of $X$, $\sigma_{\mathrm{Y}}^{2}$ the variance of $\mathrm{Y}$.

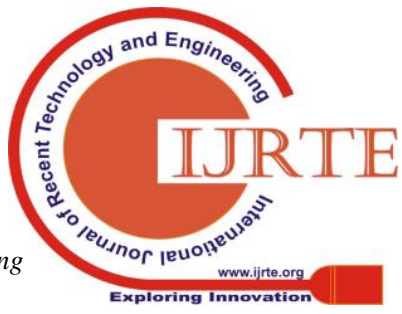




\section{A RESEARCH FOR IMPLEMENTING IMAGE INTERPOLATION USING INPAINTING AND SHEARLET TRANSFORM}

\section{CONCLUSION}

In this paper, a new sparse representation-Discrete Shearlet Transform domain inpainting model is presented. In this framework, some kinds of distinctive features are extracted from images at the encoder side, and regions with high correlation values are intentionally skipped during encoding. The remaining areas along with information associated with the edges are encoded to form the compressed output data. Removed information is to be recovered with the assistance of information sent to the decoder side. At the decoder, using a PDE-based in painting algorithm, the removed areas are recovered

\section{REFERENCES}

1. Aswathy, S.U., Glan Devadhas, G., \& Kumar, SS. (2017)Survey on Brain Tumor Detection and Segmentation Techniques on MRI Images. International Journal of Innovative Science and Research Technology, Vol. 9, No. (26), pp. 9267-9275.

2. M. Bertalmio, G. Sapiro, V. Caselles, and C. Ballester, "Image inpainting,", SIGGRAPH, pp. 1033-1038, January 2004.

3. Criminisi, A., Perez, P., Toyama, K., "Region filling and object removal by exemplar-based image inpainting.", IEEE Trans. Image Process., 13(9):1200-1212. [doi:10. 1109/TIP.2004.833105].

4. Aswathy, S.U., Glan Devadhas, G., \& Kumar, SS. (2015). Quick Detection of Brain Tumor using a Combination of EM and Levelset Method. Indian Journal of Science and Technology, Vol. 8, No. (34), pp. 1-6.

5. Patwardhan, K.A., Sapiro, G., Bertalmio, M., "Video Inpainting of Occluding and Occluded Objects.", IEEE Int. Conf. on Image Processing, 2:69-72. [doi:10.1109/ICIP. 2005.1529993].

6. Chan, T.F., Shen, J.," Image Processing and Analysis. Society for Industrial and Applied Mathematics (SIAM)", Philadelphia, p.277-279, 2005a.

7. Rane, S.D., Sapiro, G., Bertalmio, M., "Structure and texture filling-in of missing image blocks in wireless transmission and compression applications", IEEE Trans. Image Process., 12(3):296-303. [doi:10.1109/TIP.2002. 804264].

8. Aswathy, S.U., Glan Devadhas, G., \& Kumar, S.S.(2018). An improved tumor segmentation algorithm from T2 and FLAIR multimodality MRI brain images by support vector machine and genetic algorithm. Cogent Engineering, Vol. 5, No. (1) pp. 1-18

9. Wang, Z., Bovik, A.C., "Mean squared error: love it or leave it? A new look at signal fidelity measures", IEEE Signal Process. Mag., 26(1):98-117, 2009.

10. Glenn R.Easley, Demetrio Labate, and Flavia Colonna, "Shearlet based total variation diffusion for denoising", IEEE Transactions on Image Processing”, Vol.18, No.2, February,2009.

11. S.D. Rane, G. Sapiro, and M. Bertalmio, "Structure and texture filing-in of missing image blocks in wireless transmission and compression applications," IEEE Transactions on Image Processing, pp. 296-303, March 2003.

12. Aswathy, S.U., Glan Devadhas, G., \& Kumar, S.S “ Brain tumor detection and segmentation using a wrapper based genetic algorithm for optimized feature set. Cluster Computing", ISSN No. 1386-7857, pp. 1-12. 2018

13. M.Vasantha,Dr.V.Subbiah Bharathi and R.Dhamodharan, "Medical Image Feature, Extraction, Selection and Classification", International Journal of
Engineering Science and Technology, Volume 2(6), 2071-2076, 2010.

14. Awad M., Chehdi K., and Nasri A., (2007), Multicomponent image segmentation using a genetic algorithm and artificial neural network, IEEE Geoscience and remote sensing letters, 4(4), 571-575.

15. Aswathy, S.U., Glan Devadhas, G., \& Kumar, SS.\& Sreevidya, P. (2017). An Optimized Approach for SVM Based Segmentation of MR Images of Brain Tumors. Journal of Advanced Research in Dynamical and Control Systems, Vol. 9, No. (8), pp. 80-87

16. Ahmed S., Iftekharuddin K. M., and Vossough A., (2011), Efficacy of texture, shape, and intensity feature fusion for posterior-fossa tumor segmentation in MRI, IEEE Transactions on Information Technology in Biomedicine, 15(2), 206-213.

17. Chen L., Chen C. P., and Lu M., (2011), A multiplekernel fuzzy c-means algorithm for image segmentation, IEEE Transactions on Systems, Man, and Cybernetics, Part B (Cybernetics), 41(5), 1263-1274.

18. Aswathy, S.U., Glan Devadhas, G., \& Kumar, S.S. (2014). A Survey On Segmentation Of Brain Tumor From MRI Brain Images. International Journal of Applied Engineering Research, Vol 9, No. (26), pp. 9267-9275.

19. Hooda H., Verma O. P., and Singhal T., Brain tumor segmentation: A performance analysis using k-means, fuzzy c-means and region growing algorithm, In Advanced Communication Control and Computing Technologies (ICACCCT), 2014 International Conference on. IEEE, 2014, 1621-1626.

20. Moon N., Bullitt E., Van Leemput K., and Gerig G., Automatic brain and tumor segmentation, In International Conference on Medical Image Computing and Computer-Assisted Intervention. Springer, 2002, 372-379.

21. Aswathy, S.U., Glan Devadhas, G., \& Kumar, S.S (2014). A Survey On Detection Of Brain Tumor From MRI Brain Images. IEEE Digital Library, Vol. XX, No. (XX), pp. 871-877.

22. Moon N., Bullitt E., Van Leemput K., and Gerig G., Automatic brain and tumor segmentation, In International Conference on Medical Image Computing and Computer-Assisted Intervention. Springer, 2002, 372-379. 